\title{
Shear Lag Models for Discontinuous Composites: Fibre End Stresses and Weak Interface Layers
}

\author{
M.J. Starink ${ }^{\mathrm{a}, *}$ and S. Syngellakis ${ }^{\mathrm{b}}$ \\ ${ }^{a}$ Materials Research Group, School of Engineering Sciences; and \\ ${ }^{b}$ Computational Engineering and Design Research Group, School of Engineering Sciences; \\ University of Southampton, Southampton S017 1BJ, UK
}

\begin{abstract}
A new shear lag (SL) type model for stress transfer in a composite with cylindrical fibres is derived. It accounts for fibre end stresses in an approximate yet realistic manner, and leads to a new formula for predicting the Young's modulus of the composites. The predictions of this model were found to agree well with data for metal matrix composites (MMCs) with fibres of various aspect ratios. The accuracy and relative simplicity of the new model have been exploited in the development of an approximate analytical model for the stress transfer and macroscopic yield stress of a composite that contains a weak layer in the matrix adjacent to the reinforcement. With the aid of the latter model, the proof stress of MMCs which contain a precipitate free zone (PFZ) around the reinforcement, can be studied; experimental data obtained for aged $8090 \mathrm{MMCs}$ is consistent with the model predictions.
\end{abstract}

Keywords: $\quad$ shear lag model, stress transfer, MMC, PFZ, interface, Al-Li

\section{Introduction}

The elastic response of composite systems to externally applied stress can be predicted by Eshelby type models, which are valid for ellipsoidal inclusions $[1,2,3,4]$, or by shear lag (SL) type models, which have been proposed for aligned cylindrical inclusions [5,6,7,8]. Even though stress fields around cylindrical inclusions are very similar to those around ellipsoidal inclusions with the same aspect ratio [9], Eshelby type model predictions differ considerably from SL type model predictions with regard to stress transfer in short fibre and particulate composites $[5,8,10]$. This indicates that, as a result of quite crude assumptions concerning the magnitude of the stresses at the ends of the fibres, the current SL type models severely underestimate the load transfer to the reinforcement. 
Hence, Eshelby type models have tended to be favoured over SL type models. Nevertheless, SL type models offer advantages both in terms of the relative ease of the modelling and because they represent a better approximation of the often angular shapes of inclusions in various real composites.

Apart from the elastic response, also the yielding in composites can be analysed with SL and Eshelby type models. Several studies of yield strength of composites with a homogeneous matrix yield strength have been presented [11,12]. However, in the present paper, the focus is on composite systems that have a weak interfacial matrix layer around the reinforcement. These types of interfaces are for instance encountered in Al-Li and Al-Si-Mg based composites, in which a matrix layer devoid of the strengthening precipitates forms in the course of the ageing temper $[13,14,15,16]$. This type of layer in a precipitation strengthened metallic material is generally called a precipitate free zone (PFZ). The relative simplicity of SL type models can be exploited to modify them for specific situations. In the present paper, it is shown that an SL type model can be used to derive analytical expressions for stress transfer of a composite system that contains a weak matrix layer adjacent to the reinforcement.

Thus, the aim of the present paper is twofold. Firstly, it is shown that improving the description of the stresses at the fibre ends can enhance the accuracy of the shear lag model. Secondly, an SL type model is derived for the case of a composite system that contains a weak interface layer between matrix and reinforcement.

\section{A new shear lag model}

\subsection{Formulation}

According to the shear lag model (see schematic of geometry in Fig. 1), the axial stress in the cylindrical fibre is given by (see e.g. [10]):

$$
\sigma_{\mathrm{r}}(\mathrm{z})=E_{\mathrm{r}} \varepsilon_{\mathrm{c}}+A \sinh \left(\frac{\gamma z}{r_{\mathrm{o}}}\right)+B \cosh \left(\frac{\gamma z}{r_{\mathrm{o}}}\right)
$$

where $\varepsilon_{c}$ is the average macroscopic strain of the composite,

$$
\gamma=\sqrt{\frac{2 E_{\mathrm{m}}}{E_{\mathrm{r}}\left(1+v_{\mathrm{m}}\right) \ln \left(1 / f_{\mathrm{r}}\right)}}
$$

$E_{\mathrm{r}}$ and $E_{\mathrm{m}}$ are the Young's moduli of the reinforcement and the matrix, respectively, $v_{\mathrm{m}}$ is Poisson's ratio of the matrix, $f_{\mathrm{r}}$ is the volume fraction of reinforcement and $r_{\mathrm{o}}$ is the radius of the fibre crosssection. Constants $A$ and $B$ are dependent on the assumed stress at the fibre ends, $\sigma_{\mathrm{e}}$. Taking the origin at the middle of the fibre and accounting for the symmetry gives: 


$$
\sigma_{\mathrm{r}}(\mathrm{z})=E_{\mathrm{r}} \varepsilon_{\mathrm{c}}-\left(E_{\mathrm{r}} \varepsilon_{\mathrm{c}}-\sigma_{\mathrm{e}}\right) \cosh \left(\frac{\gamma z}{r_{\mathrm{o}}}\right) \operatorname{sech}\left(\gamma s_{\mathrm{r}}\right)
$$

where $s_{\mathrm{r}}$ is the aspect ratio of the fibre. Hence it follows that the stress at $z=0$ is given by:

$$
\sigma_{\mathrm{r}}(z=0)=E_{\mathrm{r}} \varepsilon_{\mathrm{c}}-\left(E_{\mathrm{r}} \varepsilon_{\mathrm{c}}-\sigma_{\mathrm{e}}\right) \operatorname{sech}\left(\gamma S_{\mathrm{r}}\right)
$$

In the literature several ways of determining $\sigma_{\mathrm{e}}$ have been proposed $[8,10,17,18]$. In the most simple version of the shear lag model, here denoted as the debonded shear lag (DSL) model, $\sigma_{\mathrm{e}}$ is simply neglected, that is, $\sigma_{\mathrm{e}}=0$. The DSL model is not reasonable for well-bonded fibres with relatively low aspect ratio, but is quite adequate for fibres with high aspect ratio. In his modification of the DSL model, Clyne [10] suggested that it is reasonable to assume $\sigma_{\mathrm{e}}$ as the average of the remote matrix stress and the peak fibre stress in the absence of fibre end stresses. It can be shown [10] that this leads to:

$$
\sigma_{\mathrm{e}}=E_{\mathrm{m}}^{\prime} \varepsilon_{\mathrm{c}}
$$

where

$$
E_{\mathrm{m}}^{\prime}=\frac{E_{\mathrm{r}}\left[1-\operatorname{sech}\left(\gamma s_{\mathrm{r}}\right)\right]+E_{\mathrm{m}}}{2}
$$

(CSL model)

From this the average longitudinal stress $\sigma_{\mathrm{c}}$ can be calculated and the corresponding composite modulus according to the CSL model is obtained as $[5,10]$ :

$$
E_{\mathrm{c}}=\frac{\sigma_{\mathrm{c}}}{\varepsilon_{\mathrm{c}}}=f_{\mathrm{r}} E_{\mathrm{r}}\left[1-\frac{E_{\mathrm{r}}-E_{\mathrm{m}}^{\prime}}{E_{\mathrm{r}}} \frac{\tanh \left(\gamma S_{\mathrm{r}}\right)}{\gamma S_{\mathrm{r}}}\right]+\left(1-f_{\mathrm{r}}\right) E_{\mathrm{m}}
$$

The latter equation gives the average stress in the composite as the weighted average of the mean stress in the reinforcement ( $1^{\text {st }}$ term on the right hand side of the latter equation) and the average stress in the matrix $\left(2^{\text {nd }}\right.$ term on the right hand side of the latter equation). It has been shown that Clyne's version of the shear lag model (CSL) is an improvement over the DSL model in terms of the prediction of Young's modulus data of Al-SiC composites. However, both versions underestimate Young's modulus of such composites [10].

In the present work it is proposed that within the framework of the shear lag model, a more consistent approach would be to take $\sigma_{\mathrm{e}}$ as the average of the average matrix stress $\left(E_{\mathrm{m}} \varepsilon_{\mathrm{c}}\right)$ and the peak fibre stress in the presence of fibre end stresses. Hence, it follows:

$$
2 \sigma_{\mathrm{e}}=E_{\mathrm{r}} \varepsilon_{\mathrm{c}}-\left(E_{\mathrm{r}} \varepsilon_{\mathrm{c}}-\sigma_{\mathrm{e}}\right) \operatorname{sech}\left(\gamma S_{\mathrm{r}}\right)+E_{\mathrm{m}} \varepsilon_{\mathrm{c}}
$$

which leads again to Eq. (5) but with $E_{\mathrm{m}}^{\prime}$ redefined as 


$$
E_{\mathrm{m}}^{\prime}=\frac{E_{\mathrm{r}}\left[1-\operatorname{sech}\left(\gamma s_{\mathrm{r}}\right)\right]+E_{\mathrm{m}}}{2-\operatorname{sech}\left(\gamma s_{\mathrm{r}}\right)}
$$

This version will be denoted as the SSSL model. Combining Eqs 7 and 9 gives:

$$
E_{\mathrm{c}}=f_{\mathrm{r}} E_{\mathrm{r}}\left[1-\left(\frac{1-E_{\mathrm{m}} / E_{\mathrm{r}}}{2-\operatorname{sech}\left(\gamma s_{\mathrm{r}}\right)}\right) \frac{\tanh \left(\gamma s_{\mathrm{r}}\right)}{\gamma \mathrm{s}_{\mathrm{r}}}\right]+\left(1-f_{\mathrm{r}}\right) E_{\mathrm{m}}
$$

Jiang et al. [8] have also proposed an SL type model based on an expression for $\sigma_{\mathrm{e}}$ which differs from the ones presented above. In the discussion that follows, this version will be referred to as the JSL model. It is further noted that the semi-empirical Tsai-Halpin equation [19] has also been used to describe the elastic response of composite systems.

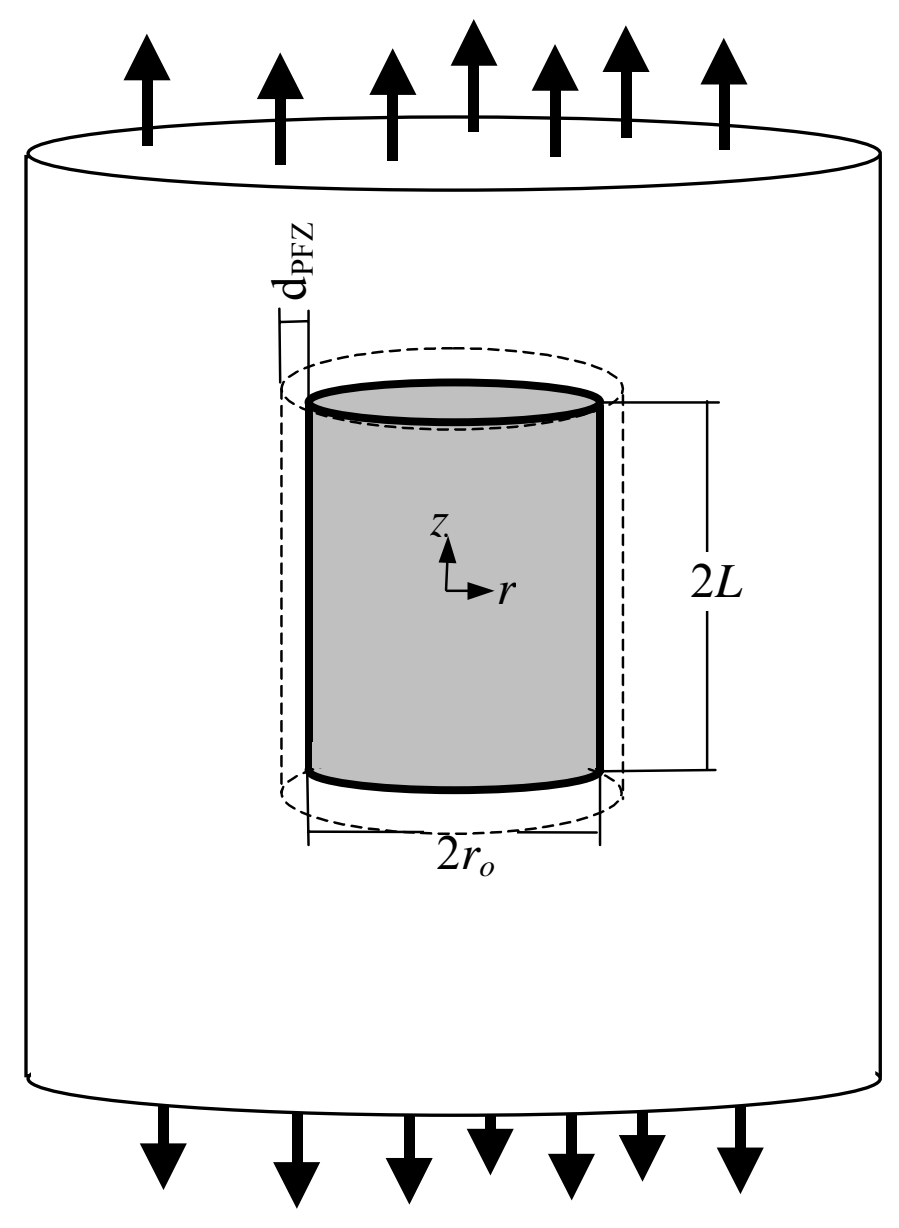

Fig. 1 Geometry of cylindrical fibre in a composite material which is subjected to external loading. The extend of the weak zone around the fibre is indicated by the dotted line. 


\subsection{Comparison with experimental data}

In comparing the predicted $E_{\mathrm{c}}$ with measured values, it is important to select only data on alloys for which the reinforcement is approximately cylindrical (or approximately ellipsoidal) with aspect ratios that have been characterised in detail. Such data is available for SiC reinforced Al-based alloys with $s_{\mathrm{r}}$ of about $2\left[20,21^{*}\right], s_{\mathrm{r}}$ of about 3 [22] and $s_{\mathrm{r}}$ of about 4 [8,23]. The predictions from the various models are compared with these data in Fig. $2\left(s_{\mathrm{r}}=2\right)$, Fig. $3\left(s_{\mathrm{r}}=3\right)$, Fig. $4\left(s_{\mathrm{r}}=4\right)$ and Fig. 5. It should be noted that $E_{\mathrm{m}}$ depends on the composition of the Al-based alloys, especially the Li content. In order to make valid comparisons for cases in which $E_{\mathrm{m}}$ varies considerably between the alloys, in some figures the ratio $E_{\mathrm{c}} / E_{\mathrm{m}}$ rather than $E_{\mathrm{c}}$ is plotted. In these cases $E_{\mathrm{m}}$ is taken as the average value for the alloys considered. The plots in Figs 2-5 show that the present SSSL model fits well to the measured data, and, in comparison with the other models, it appears to provide the best overall fit.

The relative accuracy of the model predictions depends critically on the aspect ratio of the inclusions (see Fig. 5). As a consequence, the present comparison of data and model predictions should only be considered as just an indication of the SSSL model's accuracy, since, in many real MMCs, the range of aspect ratios of the inclusions is quite broad whilst the inclusions are not perfectly aligned [22]. This point is illustrated via an investigation of $E_{c}$ data on so-called particulate reinforced, Al-based MMCs [24,25,26,27], which are technologically important because they possess relatively homogeneous properties and are relatively cheap to produce. Single particulate reinforced MMCs generally contain a range of angular particles. Within a single composite a broad range of reinforcement sizes and shapes can be encountered and aspect ratios vary between 1 and 4 [24,27]. Due to the thermo-mechanical processing (extrusion, rolling), the longer axis of the reinforcing particles (especially the ones with higher $s_{\mathrm{r}}$ ) tend to be aligned along the axis of the main working direction and, for various MMCs, the effective $s_{\mathrm{r}}$ along this axis has been estimated at 1.4 to $1.8[20,25,27,28]$. Comparison of data on $E_{\mathrm{c}}$ along the axis of the main working direction with the predictions of the models (Fig. 6, with $s_{\mathrm{r}}=1.5$ ) shows that for these composites the best fit is provided by the Eshelby model (see also Refs. [13,24,25,27]). The SSSL model, whilst showing a considerable improvement over the CSL model, underpredicts this data by about 15 to $25 \%$. In discussing this finding it is important to note that these each composite contains angular reinforcements with a broad range of shapes and aspect ratios, and, hence, the suitability of any one of the models or the adopted effective average aspect ratio may be questionable. Hence, these data do not provide a conclusive proof as to the validity of the various models, but they do indicate that at these very low aspect ratios the Eshelby model is more appropriate than the SSSL model.

\footnotetext{
* Actually, the $\mathrm{SiC}$ fibres in the $6061 \mathrm{Al}$ based composites studied by McDanels [21] have a wide range of aspect ratios. In correspondence to Clyne's assessment [10] we estimate the effective aspect ratio of the fibres in these composites at about 2. This older data is included in the present paper to facilitate comparison with Clyne's work.
} 


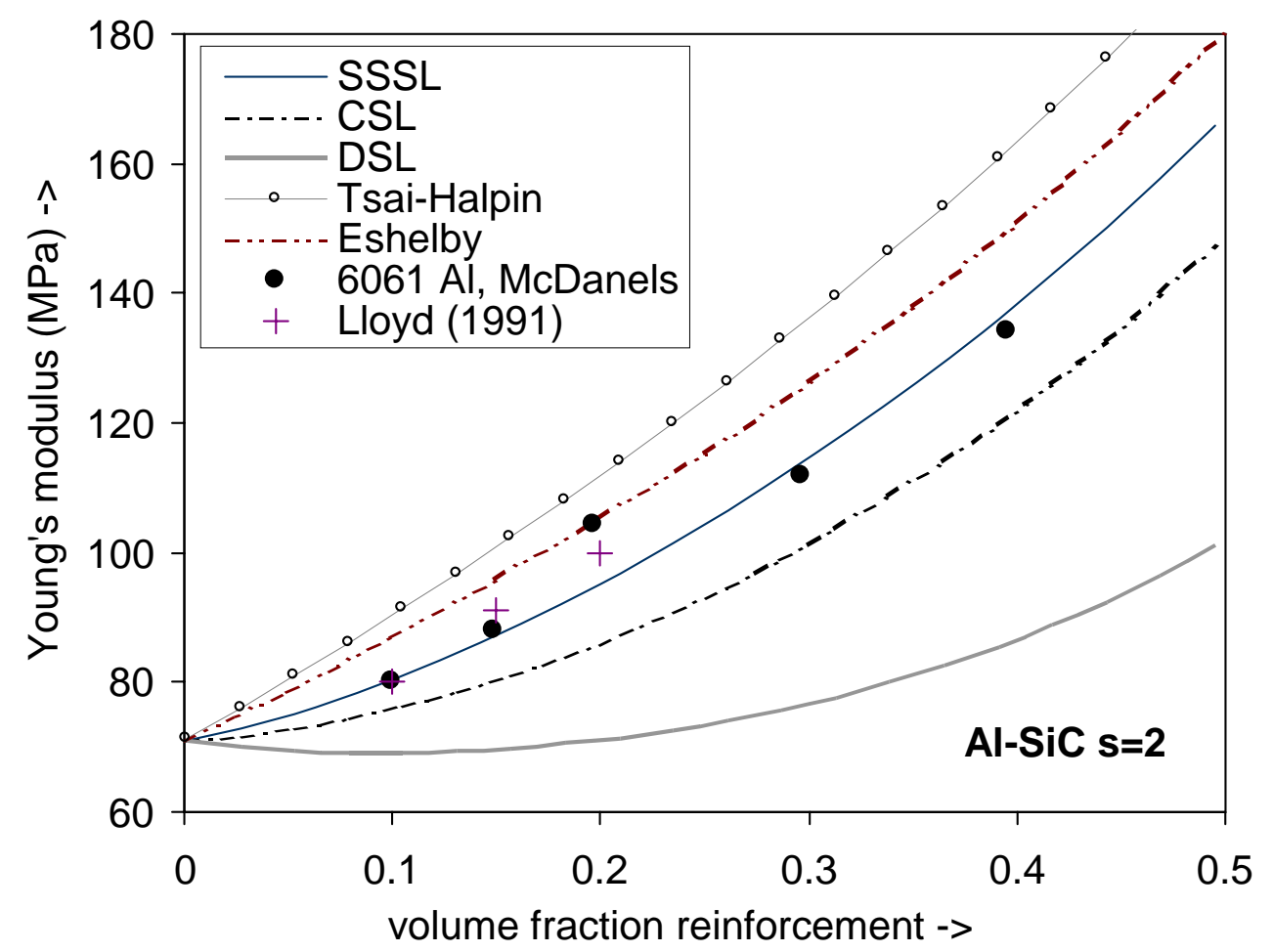

Fig. 2 Comparison of predicted and measured Young's modulus of Al-SiC composites with $s_{\mathrm{r}}=2$. $\left(E_{\mathrm{Al}}=71 \mathrm{GPa}, E_{\mathrm{SiC}}=450 \mathrm{GPa}.\right)$

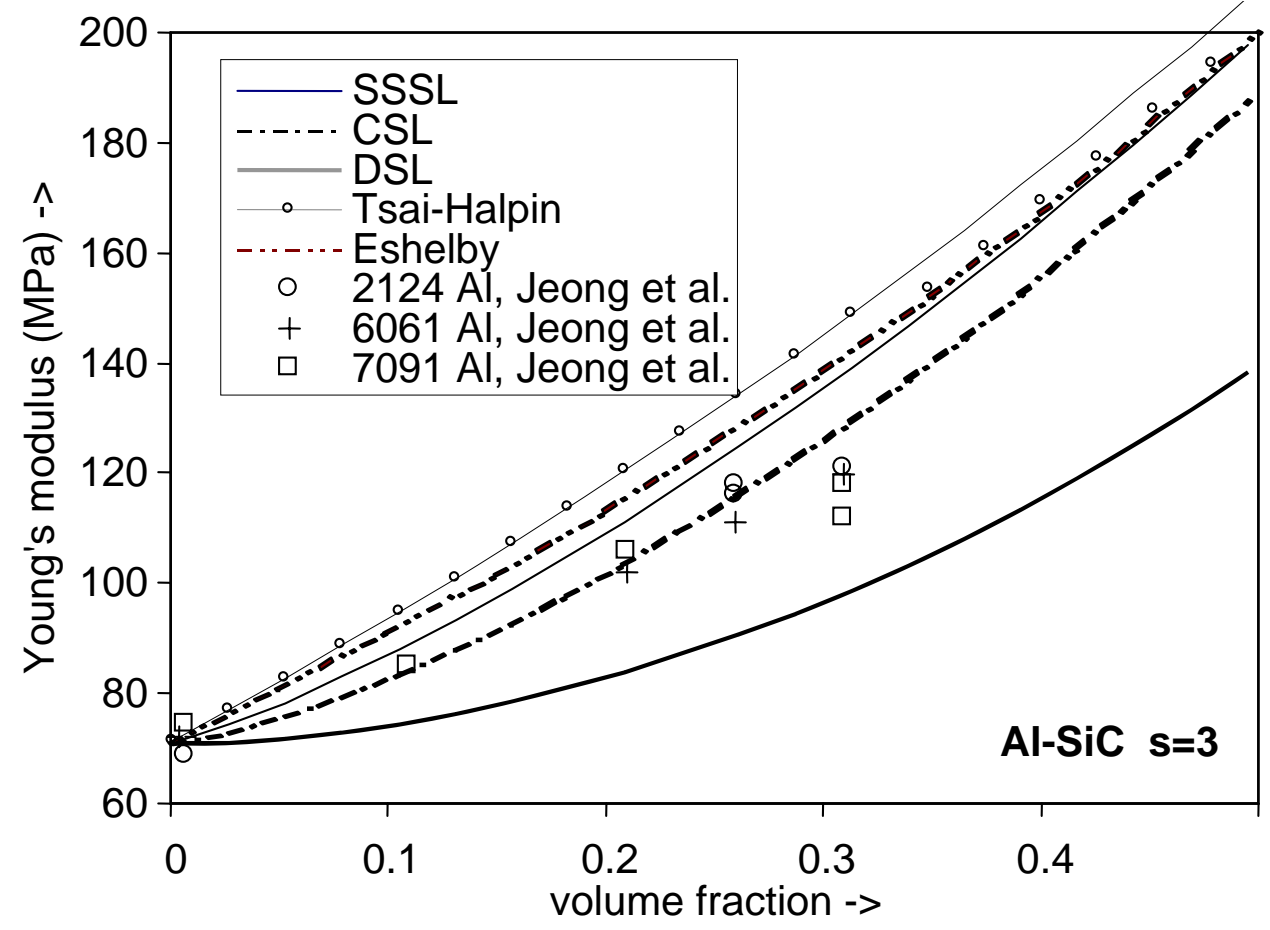

Fig. 3 Comparison of predicted and measured Young's modulus of Al-SiC composites with $s_{\mathrm{r}}=3$. $\left(E_{\mathrm{Al}}=71 \mathrm{GPa}, E_{\mathrm{SiC}}=450 \mathrm{GPa}.\right)$ 


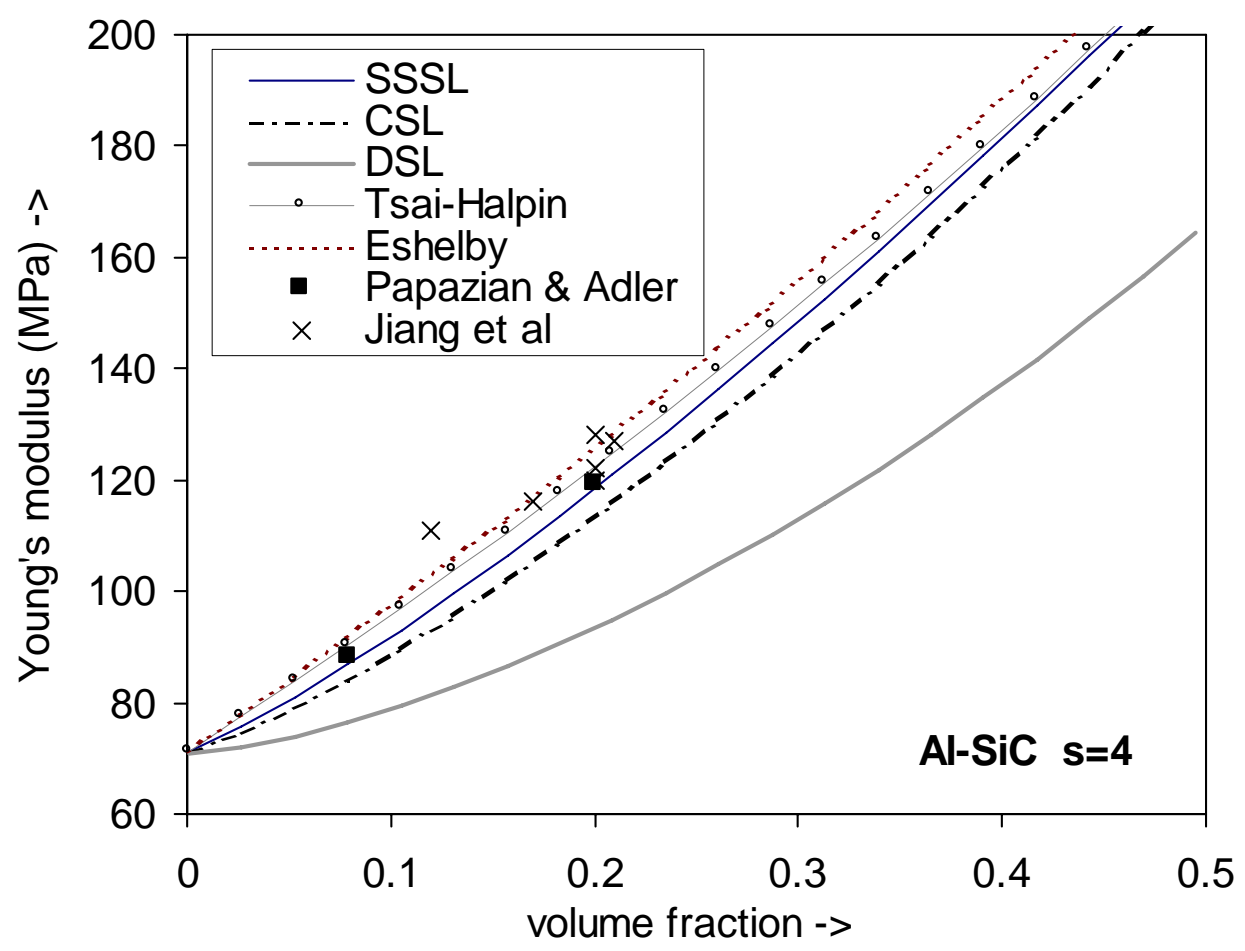

Fig. 4 Comparison of predicted and measured Young's modulus of Al-SiC composites with $s_{\mathrm{r}}=4$. $\left(E_{\mathrm{Al}}=78 \mathrm{GPa}, E_{\mathrm{SiC}}=450 \mathrm{GPa}\right.$. $)$

In more general terms the present comparison of the various models with the available data indicates that neither the Eshelby model nor any SL type model can fit the available data for all volume fractions and aspect ratios. This is thought to be mainly due to the approximations made in the development of these models (mean field assumptions in the Eshelby model, approximations for fibre end stresses in SL models), whilst inconsistency between assumed and actual shape of inclusions may also play a part. Nevertheless, in terms of predictive capability, the SSSL model is clearly a considerable improvement over other SL type models ${ }^{\dagger}$, and, on balance, this relatively simple, closed form solution appears to perform in some cases nearly as well as, and in other cases better than the Eshelby model. From a modelling perspective, it is further encouraging to note that of the four SL models considered, the SSSL model predictions are generally closest to those of the Eshelby model. Thus, the present SSSL model alleviates the problem of the large discrepancies [5,10] between previous shear lag (DSL, CSL) and Eshelby model predictions (see Fig. 5). Specifically, the SSSL model predicts $E_{\mathrm{r}} \geq E_{\mathrm{c}} \geq E_{\mathrm{m}}$ for all $s_{\mathrm{r}}$, resolving the physically unreasonable, for perfectly bonded fibres, CSL model predictions of $E_{\mathrm{c}}<E_{\mathrm{m}}, E_{\mathrm{r}}$ for low $s_{\mathrm{r}}$ (that is, for $s_{\mathrm{r}}<0.9$ in $\mathrm{Al} / \mathrm{SiC}$ composites).

\footnotetext{
$\dagger \quad$ It should also be noted that, whilst the JSL model [8] predictions for $E_{\mathrm{c}}$ appear, in general, to be less accurate than the corresponding ones by the SSSL model, the strength of the JSL model lies especially in its capability of predicting local stresses in the matrix and in assessing the effect of residual stresses on the yield behaviour of composites.
} 


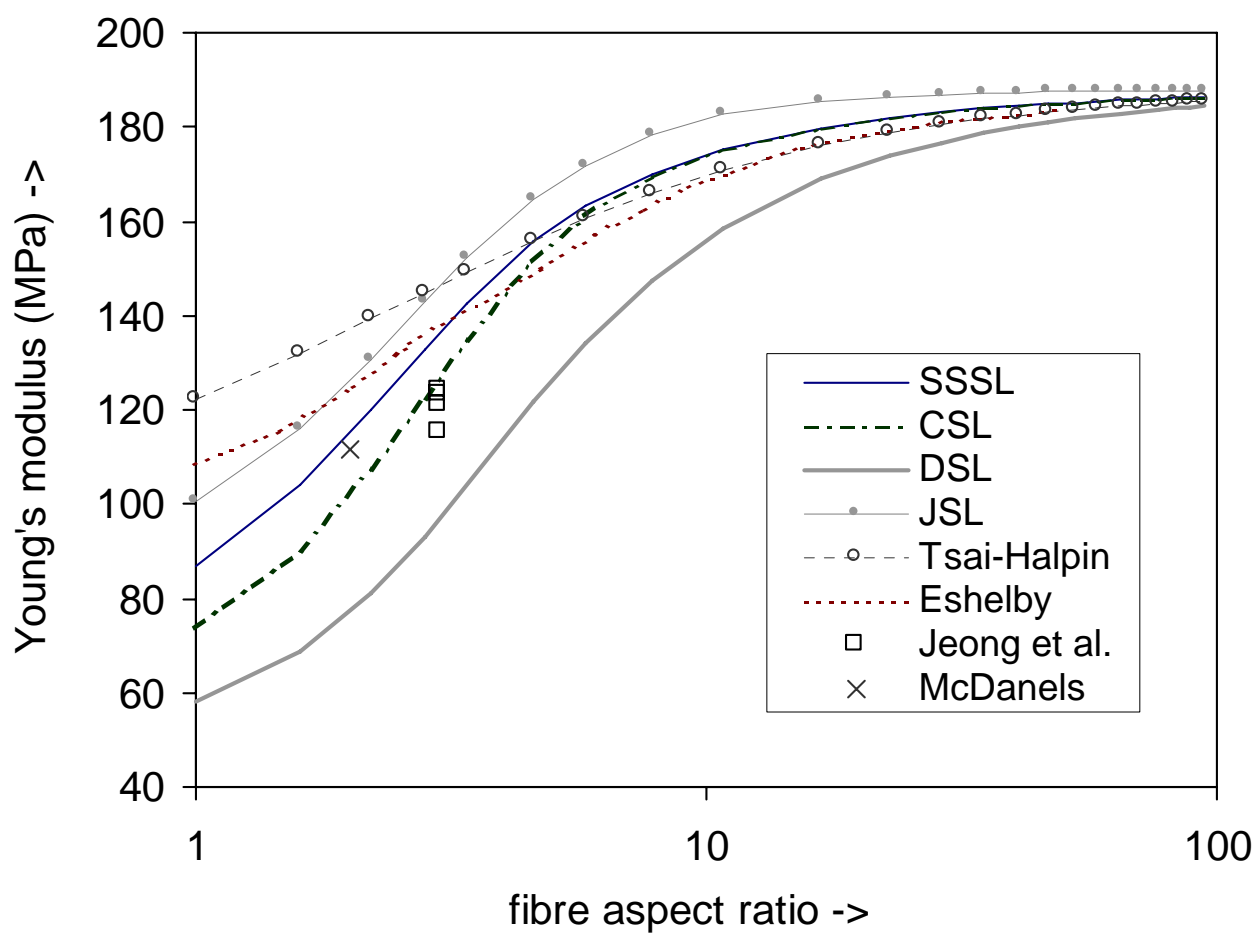

Fig. 5 Comparison of Young's modulus of Al-30vol\%SiC composites as predicted by different shear lag type models and by the Eshelby model. $\left(E_{\mathrm{Al}}=71 \mathrm{GPa}, E_{\mathrm{SiC}}=450 \mathrm{GPa}\right.$. $)$

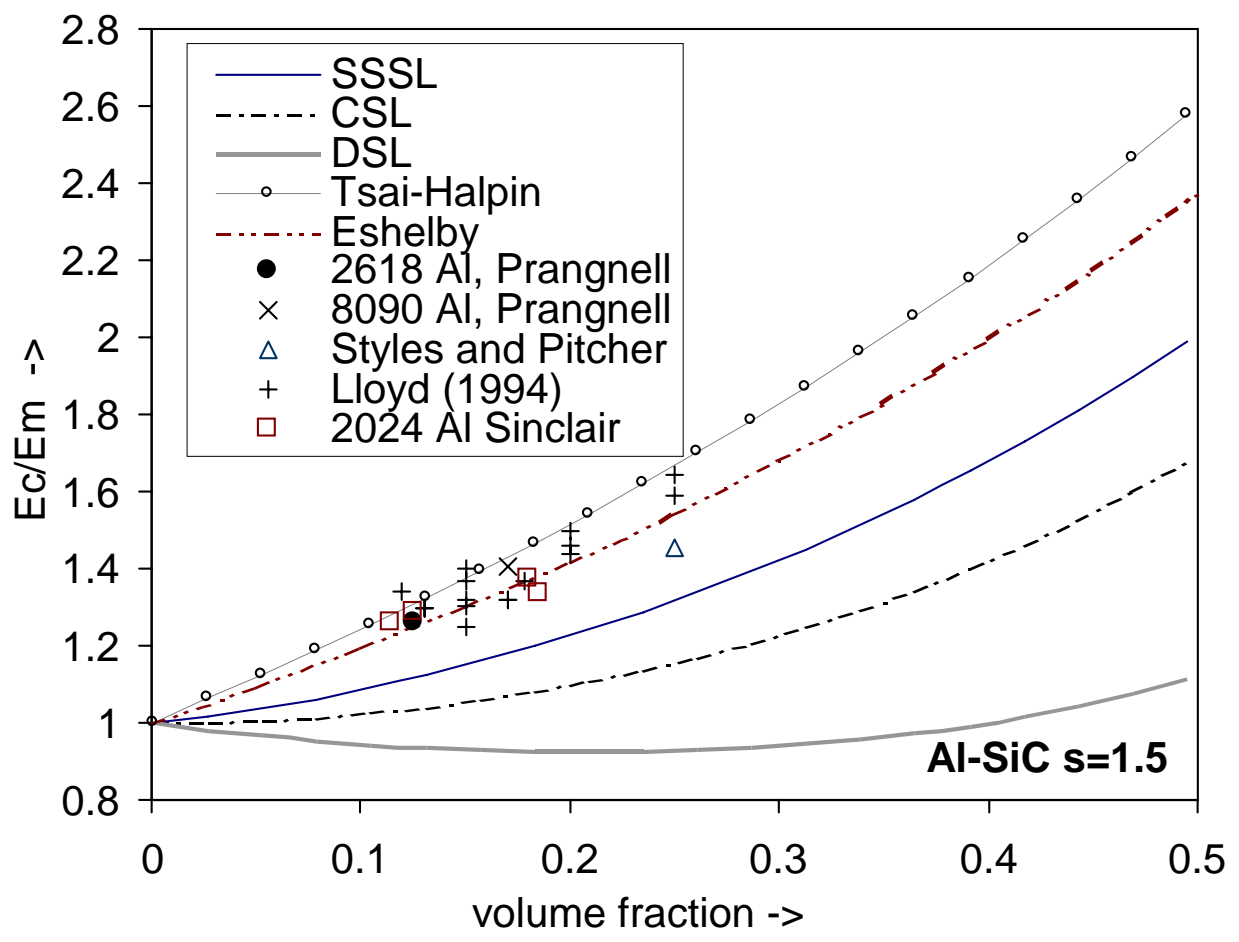

Fig. 6 Comparison of predicted and measured Young's modulus of particulate reinforced Al-SiC composites. $s_{\mathrm{r}}$ is estimated to equal 1.5. $\left(E_{\mathrm{Al}}=78 \mathrm{GPa}, E_{\mathrm{SiC}}=450 \mathrm{GPa}\right.$. $)$ 
In summarising this section, it is concluded that the present modification of the shear lag model, the SSSL model, fits well to the experimental data on fibre reinforced composites and avoids the physically unreasonable predictions made by earlier SL type models.

\section{The effect of a weak interface layer on the yield stress}

\subsection{Modelling}

The present analysis of the yield strength of a composite with a weak interface layer is based on the SSSL model described in the previous section. Although the model is described in a general manner, several assumptions and examples are chosen especially with metallic matrices reinforced by relatively stiff fibres in mind. Some simplifying assumptions are made with the aim of obtaining explicit, closed form solutions.

When a weak interface layer is present around the reinforcement, the stresses that can be sustained by the weak interface layer before local yielding occurs are limited and the stress transfer to the reinforcement will be reduced. In order to arrive at a mathematically tractable solution, first the strains at the fibre ends will be estimated in the simple case when the weak interface layer is very weak in comparison to the matrix $\left(\sigma_{\mathrm{y}}^{\mathrm{PFZ}}<<\sigma_{\mathrm{y}}^{\mathrm{m}}\right)$. The fibres are assumed much stiffer than the matrix $\left(E_{\mathrm{r}} \gg E_{\mathrm{m}}\right)$. Hence, the elastic deformation of the fibres is negligibly small and the composite deforms via deformation of matrix and weak interface layer only. Thus, the elongation of the weak interface layer at the fibre ends should be compatible with that of the matrix at some perpendicular distance from the fibre where matrix strain is approximately equal to composite strain. This condition gives:

$$
\left(1+\varepsilon_{\mathrm{e}}\right) d_{\mathrm{PFZ}}+L \approx\left(1+\varepsilon_{\mathrm{c}}\right)\left(L+d_{\mathrm{PFZ}}\right)
$$

where $d_{\mathrm{PFZ}}$ and $\varepsilon_{e}$ are, respectively, the thickness and strain of the layer, $L$ is the half-length of the fibre (Fig. 1) and $\varepsilon_{c}$ the composite strain. From (11a), it follows:

$$
\varepsilon_{\mathrm{e}} \approx \varepsilon_{\mathrm{c}}\left[\frac{L}{d_{\mathrm{PFZ}}}+1\right]
$$

Since $d_{\mathrm{PFZ}}$ is much smaller than $L$, it is evident from Eq. $11 \mathrm{~b}$ that the maximum stress at the fibre ends in the presence of a weak interface layer will reach the yield strength of that layer, $\sigma_{y}^{P F Z}$, well before significant yielding throughout the remainder of the matrix of the composite occurs. As a consequence, the stress transfer due to stresses at the fibre ends is essentially reduced by a factor $\sigma_{y}^{P F Z} / \sigma_{e}$, in comparison to a composite with homogeneous matrix strength. 
Apart from the stresses at the fibre ends, the stresses at the sides of the fibre will also be influenced by the presence of the weak interfacial layer, and, especially for longer fibres, the shear stresses in the yielding layer adjacent to the fibre sides will be important in determining the overall stress response of the composites. An indication of this influence is provided by the SL prediction of the interfacial shear stress distribution along the fibre side. This shear reaches a maximum near the fibre ends and the composite strain initiating yield locally is found equal to

$$
\varepsilon_{\mathrm{c}}^{*}=\frac{\sigma_{\mathrm{y}}^{\mathrm{PFZ}}}{E_{\mathrm{r}}}\left(1+\frac{1}{\gamma \tanh \left(\gamma s_{\mathrm{r}}\right)}\right)
$$

Since $E_{\mathrm{r}}$ is much greater than $E_{\mathrm{m}}$ and $\sigma_{\mathrm{y}}^{\mathrm{PFZ}}$ is smaller than the matrix yield stress $\sigma_{\mathrm{y}}^{\mathrm{m}}$, it is clear from the latter equation that, for fibres with large aspect ratio, yielding of the interface layer on the fibre side will start well before the matrix yields. In order to generate a simple mathematical model for this gradual weakening process, the assumption is here made that the average fibre stress is reduced by the factor $\sigma_{\mathrm{y}}^{\mathrm{PFZ}} / \sigma_{\mathrm{e}}$ where $\sigma_{\mathrm{e}}$ is the fibre end stress for non-yielding matrix at identical $\sigma_{\mathrm{c}}$. This assumption leads to the following expression for the overall stress in the composite:

$$
\frac{\sigma_{\mathrm{c}}}{\varepsilon_{\mathrm{c}}}=f_{\mathrm{r}} E_{\mathrm{r}}\left(1-\frac{E_{\mathrm{r}}-E_{\mathrm{m}}^{\prime}}{E_{\mathrm{r}}} \frac{\tanh \left(\gamma S_{\mathrm{r}}\right)}{\gamma S_{\mathrm{r}}}\right) \frac{\sigma_{\mathrm{y}}^{\mathrm{PFZ}}}{\sigma_{\mathrm{e}}}+\left(1-f_{\mathrm{r}}\right) E_{\mathrm{m}}
$$

As indicated by Eqs. 3 and 4, for fibres with a small aspect ratio, the stress transfer at the fibre ends is the dominant factor in determining the overall response. Thus, the average fibre stress is very close to $\sigma_{\mathrm{e}}$, therefore the weak interface layer will only yield at the fibre end face and Eq. 13 can be applied without significant loss of accuracy. In contrast, for fibres with a large aspect ratio, there is considerable stress transfer remaining at the side of the fibre even after yielding of the weak layer has occurred at the fibre ends. The present treatment essentially approximates the weakening of this stress transfer due to the presence of the weak layer by assuming that the shear stress averaged over one half of the fibre is proportional to $\sigma_{\mathrm{e}}$. The proposed approximation may, however, become less accurate with increasing aspect ratio.

As the total volume of the weak interface layer will generally be very small, the yielding in the weak interface layers will not lead to a major deviation from linearity of the stress-strain curve of the composite, that is, on a macroscopic scale, the yielding in the weak interface layers will be barely detectable. Significant deviation from linearity will occur when yielding occurs in the remainder of the matrix, that is, when the average stress in the matrix exceeds the matrix yield stress (see also [8]). Hence, it follows:

$$
\sigma_{\mathrm{y}}^{\mathrm{c}}=\varepsilon_{\mathrm{c}} f_{\mathrm{r}} E_{\mathrm{r}}\left(1-\frac{E_{\mathrm{r}}-E_{\mathrm{m}}^{\prime}}{E_{\mathrm{r}}} \frac{\tanh \left(\gamma S_{\mathrm{r}}\right)}{\gamma S_{\mathrm{r}}}\right) \frac{\sigma_{\mathrm{y}}^{\mathrm{PFZ}}}{\sigma_{\mathrm{e}}}+\left(1-f_{\mathrm{r}}\right) \sigma_{\mathrm{y}}^{\mathrm{m}}
$$


The strain in the composite may be further approximated at the yield point by $\varepsilon_{\mathrm{c}} \cong \sigma_{\mathrm{y}}^{\mathrm{m}} / E_{\mathrm{m}}$ and, hence, Eq. 14 can be rearranged to give a linear relationship between the macroscopic yield strength of the composite and the yield strength of the matrix:

$$
\frac{\sigma_{\mathrm{y}}^{\mathrm{c}}}{\sigma_{\mathrm{y}}^{\mathrm{m}}} \cong f_{\mathrm{r}}\left(\frac{E_{\mathrm{r}}}{E_{\mathrm{m}}}-\frac{E_{\mathrm{r}}-E_{\mathrm{m}}^{\prime}}{E_{\mathrm{m}}} \frac{\tanh \left(\gamma s_{\mathrm{r}}\right)}{\gamma s_{\mathrm{r}}}\right) \frac{\sigma_{\mathrm{y}}^{\mathrm{PFZ}}}{\sigma_{\mathrm{e}}}+\left(1-f_{\mathrm{r}}\right)
$$

or, by using Eq. 5:

$$
\frac{\sigma_{\mathrm{y}}^{\mathrm{c}}}{\sigma_{\mathrm{y}}^{\mathrm{m}}} \cong f_{\mathrm{r}}\left(\frac{E_{\mathrm{r}}}{E_{\mathrm{m}}^{\prime}}-\frac{E_{\mathrm{r}}-E_{\mathrm{m}}^{\prime}}{E_{\mathrm{m}}^{\prime}} \frac{\tanh \left(\gamma S_{\mathrm{r}}\right)}{\gamma S_{\mathrm{r}}}\right) \frac{\sigma_{\mathrm{y}}^{\mathrm{PFZ}}}{\sigma_{\mathrm{y}}^{\mathrm{m}}}+\left(1-f_{\mathrm{r}}\right)
$$

It is noted that when $\sigma_{\mathrm{y}}^{\mathrm{PFZ}} / \sigma_{\mathrm{e}}$ equals 1 , it follows from Eqs. 7 and 15 that $\sigma_{\mathrm{y}}^{\mathrm{c}} / \sigma_{\mathrm{y}}^{\mathrm{m}}=E_{\mathrm{c}} / E_{\mathrm{m}}$. This case however represents an unrealistic situation of no local matrix yielding over the reinforcementmatrix interface. Hence the composite behaves linearly until overall yield which coincides with matrix yield, that is, at strain $\varepsilon_{c}=\frac{\sigma_{y}^{c}}{E_{c}}=\frac{\sigma_{y}^{m}}{E_{m}}$. Eq. 16 shows that $\sigma_{\mathrm{y}}^{\mathrm{c}} / \sigma_{\mathrm{y}}^{\mathrm{m}} \neq E_{\mathrm{c}} / E_{\mathrm{m}}$ when $\sigma_{\mathrm{y}}^{\mathrm{PFZ}} / \sigma_{\mathrm{y}}^{\mathrm{m}}=1$, that is, in the absence of a weak interface layer.

In most real composites, the PFZ will strain harden during plastic deformation and for this effect to be accounted for by the proposed model, the stress at the fibre ends can be calculated from a theoretical expression of strain hardening [29,30]:

$$
\sigma_{\mathrm{y}}^{\mathrm{PFZ}}=\sigma_{\mathrm{y}, 0}^{\mathrm{PFZ}}+K \sqrt{\varepsilon_{\mathrm{e}}}
$$

where $\sigma_{\mathrm{y}, 0}^{\mathrm{PFZ}}$ is the yield strength of the weak interface layer at zero pre-strain, $\varepsilon_{e}$ is the strain in the plastic zone calculated using Eq. 11 and $K$ is a parameter which depends on the strain hardening capability of the PFZ (for $8090 \mathrm{~K}$ is estimated at about $150 \mathrm{MPa}$ ).

\subsection{Verification and application}

The present model of MMCs with a weak interface region adjacent to the particles should be valid for various heat treatable Al-based MMCs in which a PFZ around the reinforcement develops. An example of such a composite is the 8090 (Al-Li-Cu-Mg) alloy reinforced with $\mathrm{SiC}$ particles. The matrix of this composite is strengthened by GPB zones, $\mathrm{S}^{\prime}\left(\mathrm{Al}_{2} \mathrm{CuMg}\right)$ and $\delta^{\prime}\left(\mathrm{Al}_{3} \mathrm{Li}\right)$ precipitates $[14,31,33,32]$, but a layer devoid of the strengthening $\delta^{\prime}\left(\mathrm{Al}_{3} \mathrm{Li}\right)$ precipitates forms around the $\mathrm{SiC}$ particles as a result of $\mathrm{Li}$ depletion of the zone caused by the formation of $\mathrm{Li}$ containing precipitates on the SiC/matrix interface [14]. The SiC particles are angular, and, although they are clearly not cylindrical, SL type models with cylindrical inclusions should provide a better approximation of the stress transfer as compared to the Eshelby type model with ellipsoidal inclusions. In a recent study [33] of the proof strengths of various Al-Li-Cu-Mg based composites and monolithic alloys, a 
comprehensive model for the strength of the matrices of these alloys and composites based on a detailed assessment of the microstructures is presented and validated. The values of $\sigma_{\mathrm{y}}^{\mathrm{m}}$ and $\sigma_{\mathrm{y}}^{\mathrm{PFZ}}$ obtained in that study are used to validate the model presented in this section of the present work. It should be noted that obtaining $\sigma_{\mathrm{y}}^{\mathrm{m}}$ and $\sigma_{\mathrm{y}}^{\mathrm{PFZ}}$ for heat treatable, PFZ-containing MMCs is not a straightforward matter, because both these strengths vary with ageing time and cannot be measured directly.

Account should also be taken of the fact that, due to the high load transfer to the reinforcement resulting from plastic deformation, MMCs have a high work hardening rate, and consequently the $(0.2 \%)$ proof stress can be considerably higher than the yield stress. In order to account for this "plastic stress transfer", the corresponding strength increment, $\Delta \sigma_{\text {pst }}$, as predicted by the Eshelbytype model presented in Ref. [34], is used. The latter work shows that $\Delta \sigma_{\mathrm{pst}}$ depends on $f_{\mathrm{r}}, s_{\mathrm{r}}$, elasticity constants and the plastic strain of the composite $\varepsilon_{\mathrm{e}}^{\mathrm{p}}$, and is estimated at about $20 \mathrm{MPa}$ for the material data of the tested MMCs considered here (Table 1). For the composites with a weak interface layer, the "plastic stress transfer" is taken account of by assuming that, similarly to the elastic stress transfer, the effect is proportional to $\sigma_{\mathrm{y}}^{\mathrm{PFZ}}$. Thus, Eq. 16 is modified to:

$$
\sigma_{0.2}^{\mathrm{c}} \cong\left[f_{\mathrm{r}}\left(\frac{E_{\mathrm{r}}}{E_{\mathrm{m}}^{\prime}}-\frac{E_{\mathrm{r}}-E_{\mathrm{m}}^{\prime}}{E_{\mathrm{m}}^{\prime}} \frac{\tanh \left(\gamma S_{\mathrm{r}}\right)}{\gamma S_{\mathrm{r}}}\right) \sigma_{\mathrm{y}}^{\mathrm{m}}+\Delta \sigma_{\mathrm{pst}}\right] \frac{\sigma_{\mathrm{y}}^{\mathrm{PFZ}}}{\sigma_{\mathrm{y}}^{\mathrm{m}}}+\left(1-f_{\mathrm{r}}\right) \sigma_{\mathrm{y}}^{\mathrm{m}}
$$

The results of the assessment of the $0.2 \%$ proof stress, presented in Table 1 , show that the proposed model predicts the proof stresses of the 8090 MMC samples quite well. In particular, it accurately predicts that, for this composite, the potential strengthening effect due the $\mathrm{SiC}$ is reduced to zero as a result of the presence of the PFZ.

Table 1 Yield strengths of $8090 / 17 \mathrm{vol} \% \mathrm{SiC}$ composites after ageing for several times at $170^{\circ} \mathrm{C}$. $\sigma_{\mathrm{y}}^{\mathrm{m}}$ and $\sigma_{\mathrm{y}}^{\mathrm{PFZ}}$ are obtained from a quantitative analysis microstructure in combination with a comprehensive model of the strengthening of Al-Li-Cu-Mg alloys [33] $\left(s_{\mathrm{r}}=1.5\right)$.

\begin{tabular}{|c|c|c|c|c|c|c|}
\hline (h) & $\begin{array}{l}d_{\mathrm{PFZ}} \\
(\mu \mathrm{m}) \\
\end{array}$ & $\begin{array}{c}\sigma_{\mathrm{y}}^{\mathrm{m}} \\
(\mathrm{MPa}) \\
\end{array}$ & $\begin{array}{c}\sigma_{\mathrm{y}}^{\mathrm{PFZ}} \\
(\mathrm{MPa}) \\
\end{array}$ & $\begin{array}{c}\sigma_{\mathrm{y}}^{\mathrm{m}}: \sigma_{\mathrm{y}}^{\mathrm{c}} \\
\text { (predicted) } \\
(\mathrm{MPa})\end{array}$ & $\begin{array}{c}\sigma_{0.2}^{\mathrm{c}} \\
\text { (predicted) } \\
(\mathrm{MPa}) \\
\end{array}$ & $\begin{array}{c}\sigma_{0.2}^{\mathrm{c}} \\
\text { (measured) } \\
(\mathrm{MPa})\end{array}$ \\
\hline 0 & 0 & 136 & - & 1.18 & 185 & 220 \\
\hline 0.17 & 0.02 & 294 & 161 & 0.95 & 297 & 306 \\
\hline 7 & 0.06 & 353 & 186 & 0.94 & 352 & 368 \\
\hline 168 & 0.12 & 399 & 243 & 0.96 & 402 & 418 \\
\hline
\end{tabular}




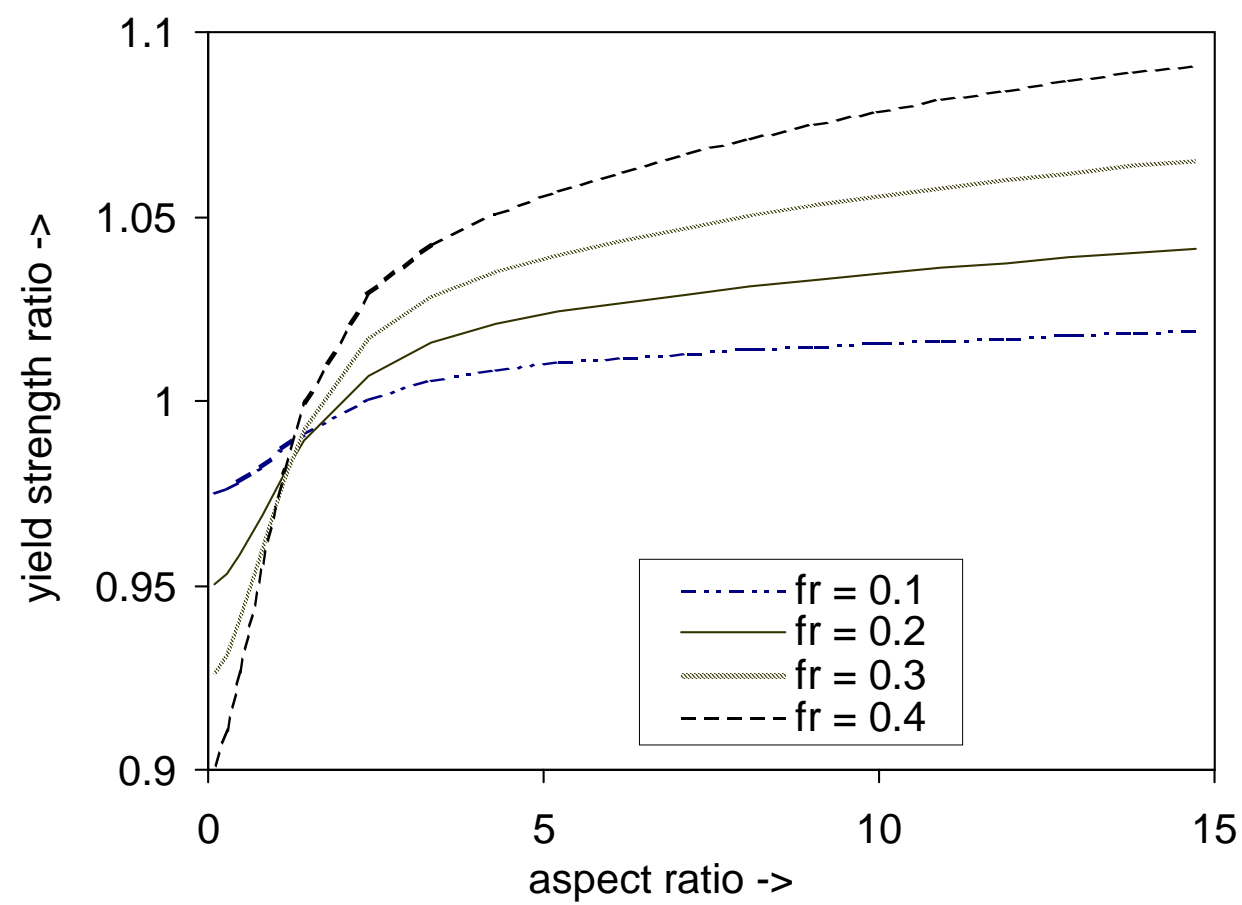

Fig. 7 The ratio of the yield strength of Al-SiC composites to the yield strength of the matrix for $\sigma_{\mathrm{y}}^{\mathrm{PFZ}} / \sigma_{\mathrm{y}}^{\mathrm{m}}=0.75$, vs. the aspect ratio of the cylindrical $\mathrm{SiC}$ reinforcement. $\left(E_{\mathrm{Al}}=71 \mathrm{GPa}\right.$, $E_{\mathrm{SiC}}=450 \mathrm{GPa}$.)

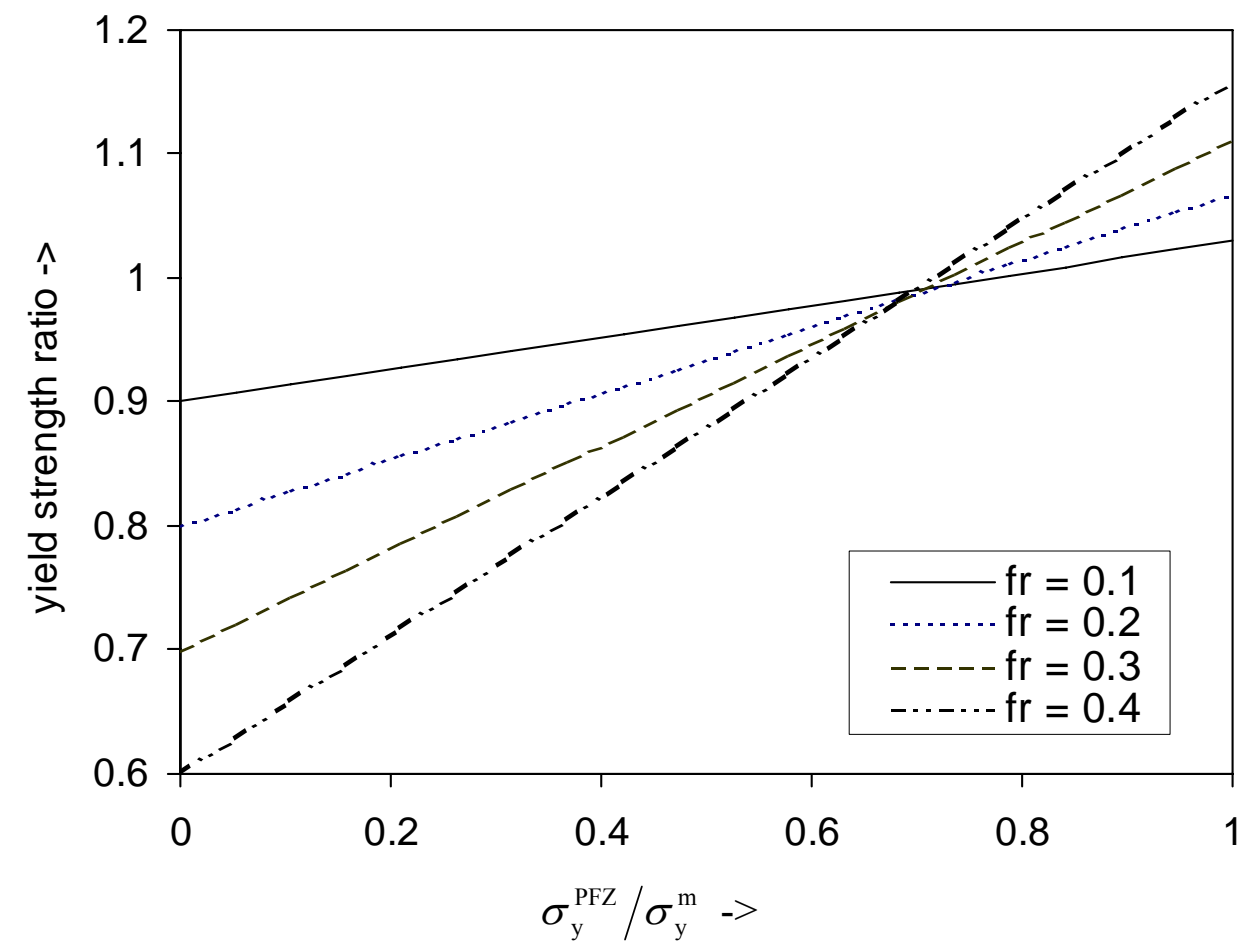

Fig. 8 The ratio of the yield strength of Al-SiC composites to the yield strength of the matrix vs. $\sigma_{\mathrm{y}}^{\mathrm{PFZ}} / \sigma_{\mathrm{y}}^{\mathrm{m}}$. The aspect ratio of cylindrical $\mathrm{SiC}$ inclusions is $2 .\left(E_{\mathrm{Al}}=71 \mathrm{GPa}, E_{\mathrm{SiC}}=\right.$ $450 \mathrm{GPa}$.) 
The above analytical expressions can be readily evaluated for any composite and selected results for the case of Al-SiC composites are presented in Fig. 7 and Fig. 8. For the sake of clarity of presentation the strain hardening of the PFZ has been neglected, that is, a perfectly plastic matrix is considered. The present simplified model predicts that for a perfectly plastic matrix, the thickness of the PFZ, $d_{\mathrm{PFZ}}$, does not influence the yield strength of the composite.

Fig. 7 shows that even for a relatively limited softening of the interface layer $\left(\sigma_{\mathrm{y}}^{\mathrm{PFZ}} / \sigma_{\mathrm{y}}^{\mathrm{m}}=0.75\right)$ the strengthening of the material as a result of the addition of fibres is predicted to be significantly reduced. This effect is especially marked for low aspect ratios of "reinforcement". Fig. 8 further illustrates the effect of a combination of a weak interface layer and low fibre aspect ratio. This figure shows that for composites with inclusions of fixed aspect ratio $\left(s_{\mathrm{r}}=2\right)$, strengthening due to the $\mathrm{SiC}$ is only achieved if $\sigma_{\mathrm{y}}^{\mathrm{PFZ}} / \sigma_{\mathrm{y}}^{\mathrm{m}}$ is larger than a critical value (for $s_{\mathrm{r}}=2$ this critical value is $0.7)$.

Having successfully applied the present model for a composite with a weak interface layer to data on one specific MMC, it is clear that more work on these type of systems is needed to obtain a full assessment of the accuracy of the model. Especially data on the effect of variation of the aspect ratio of the fibres would enhance understanding of the stress transfer in these composites with a weak interface layer. This kind of understanding can be achieved by finite element modelling, which, in addition, will lead to a detailed examination of the localised microscopic yield behaviour of the weak interface.

\section{Summary}

A new version of the shear lag (SL) model for bonded fibres has been derived. This new version (SSSL) accounts for fibre end stresses in an approximate yet realistic manner. It is shown that the SSSL model agrees well with experimental data and resolves some of the discrepancies between Eshelby type models and previous SL type models.

The accuracy and relative simplicity of the SSSL model have been exploited to derive an analytical model for the stress transfer in a composite that contains a weak layer in the matrix adjacent to the reinforcement. Evaluation of the equations for the example of an Al-SiC metal matrix composite shows that a relatively small strength differential of the weak layer with the rest of the matrix can significantly reduce the apparent macroscopic yield strength of the composite. The model predictions are consistent with yield strength measurements of aged 8090 (Al-Li-Cu-Mg) MMC samples. 


\section{Acknowledgements}

Dr. I. Sinclair's contributions in assisting with model calculations and stimulating discussions are gratefully acknowledged.

\section{References}

1 J.D. Eshelby, Proc. Roy. Soc. Lond. A 252 (1959) 561

2 T. Tanaka, K. Wakashima and T. Mori, J. Mech. Phys. Solids 21 (1973) 207

3 P.J. Withers, W.M. Stobbs and O.B. Pedersen, Acta Metall. 37 (1989) 3061

4 R.J. Arsenault and M. Taya, Acta Metall. 35 (1987) 651

5 H.L. Cox, Br. J. Appl. Phys. 3 (1952) 72.

$6 \quad$ A. Kelly and K.N. Street, Proc. R. Soc. Lond. A 328 (1972) 283.

7 D. Hull and T.W. Clyne, in 'An introduction to Composite Materials', 2nd ed., Cambridge University Press, Cambridge, U.K., 1996.

8 Z. Jiang, J. Lian, D. Yang and S. Dong, Mater. Sci. Eng. A 248 (1998) 256.

9 P.J. Withers, E.M. Chorley and T.W. Clyne, Mater. Sci. Eng. A 135 (1991) 173

10 T.W. Clyne, Mater. Sci. Eng A 122 (1989) 183

11 A. Levy and J.M. Papazian, Acta Metall. 39 (1991) 2255.

12 I. Dutta, J.D. Simms and D.M. Seigenthaler, Acta Metall. Mater. 41 (1993) 885.

13 I. Sinclair and P.J. Gregson, Mater. Sci. Techn. 13 (1997) 709.

14 M.J. Starink and P.J. Gregson, Mater. Sci. Eng. A 211 (1996) 54; M.J. Starink, P. Wang, I. Sinclair and P.J. Gregson, Acta Mater., 47 (1999) 3841.

15 B. Mingler and H.P. Karnthaler, Z. Metallkd. 84 (1993) 313

16 H. Toda, T. Kobayashi and N. Inoue, J. Jpn. Inst. Metals. 61 (1997) 120

17 C.-H. Hsueh, J. Mater. Sci. 24 (1989) 4475

18 C.-H. Hsueh, J. Mater. Sci. 30 (1995) 219

19 J.C. Halpin and S.W. Tsai, "Environmental Factors in Composite Design", Report AFML-TR-67423, US Air Force Materials Laboratory, Ohio, USA, 1967

20 D.J. Lloyd, Acta Metall. Mater., 39 (1991) 59

21 D.L. McDanels, Metall. Trans. A16 (1985) 1105

22 H. Jeong, D.K. Hsu, R.E. Shannon and P.K. Liaw, Metall. Mater. Trans. A 25 (1994) 799

23 J.M. Papazian and P.N. Adler, Metall. Trans. A 21 (1990) 401

24 C.M. Styles, $\mathrm{PhD}$ thesis, University of Southampton, 1993

25 P.B. Prangnell, T. Downes, W.M. Stobbs and P.J. Withers, Acta Mater. 42 (1994) 3425

26 C.M. Styles and P.D. Pitcher, Mater. Sci. Tech. 14 (1998) 913

27 D.J. Lloyd, Intern. Mater. Rev. 39 (1994) 1

28 I. Sinclair, unpublished work, University of Southampton, 1998

29 P. Gomiero, Y. Brechet, F. Louchet, A. Tourabi and B. Wack, Acta Metall. Mater. 40 (1992) 857.

30 M.F. Ashby, Phil. Mag. 14 (1966) 1157.

31 M.J. Starink and P.J. Gregson, Scr. Metall. Mater. 33 (1995) 893.

32 M.J. Starink, A.J. Hobson and P.J. Gregson, Scr. Metall. Mater. 34 (1996) 1711.

33 M.J. Starink, P. Wang, I. Sinclair and P.J. Gregson, Acta Mater., 47 (1999) 3855.

34 T.W. Clyne and P.J. Withers, in "An Introduction to Metal Matrix Composites", Cambridge University Press, Cambridge, U.K., 1993. 